\title{
The Outward Diffusion of Sb during Nanowire Growth Studied by Quantitative High-Angle Annular Dark Field Scanning Transmission Electron Microscopy.
}

\author{
H. Kauko ${ }^{1}$, T. Grieb ${ }^{2}$, A. M. Munshi ${ }^{3}$, K. Müller ${ }^{2}$, A. Rosenauer ${ }^{2}$, B. O. Fimland ${ }^{3}$ and A. T. J. van \\ Helvoort ${ }^{1}$. \\ ${ }^{1 .}$ Department of Physics, Norwegian University of Science and Technology (NTNU), Trondheim, \\ Norway. \\ 2. Institut für Festkörperphysik, Universität Bremen, Bremen, Germany. \\ ${ }^{3 .}$ Department of Electronics and Telecommunications, Norwegian University of Science and \\ Technology (NTNU), Trondheim, Norway.
}

Planar antimony $(\mathrm{Sb})$ containing compound semiconductors are used in a broad range of optoelectronic devices. The optical properties of these devices can be tailored by the $\mathrm{Sb}$ concentration. In the case of $\mathrm{Sb}$ containing nanowires (NWs) the high surface-to-volume ratio can affect the Sb composition control during the NW growth. In previous studies on both Au- and Ga-assisted molecular beam epitaxy (MBE) grown $\mathrm{GaAs}_{1-\mathrm{y}} \mathrm{Sb}_{\mathrm{y}}$ inserts in GaAs NWs, a reduction in the $\mathrm{Sb}$ concentration towards the outer surface was found $[1,2]$. The radial composition variations could be caused by i) As-Sb exchange at the surface and/or ii) radial GaAs overgrowth during NW growth.

In order to understand the origin for the observed radial compositional variations, we have systematically studied the radial $\mathrm{Sb}$ distribution in MBE grown Ga-assisted $\mathrm{GaAs}_{1-\mathrm{y}} \mathrm{Sb}_{\mathrm{y}} \mathrm{NWs}$ as well as in axial $\mathrm{GaAs}_{1-\mathrm{y}} \mathrm{Sb}_{\mathrm{y}}$ inserts (50-150 nm long) in both Ga- and Au-assisted GaAs NWs (Fig. 1). The Sb distribution was studied by quantitative high-angle annular dark field scanning transmission electron microscopy (HAADF STEM) on as-received NWs, i.e., no additional specimen preparation was applied. All experiments were performed on a non-corrected TEM/STEM at medium magnifications.

The experimental HAADF STEM intensities were normalized to the incident beam intensity to allow for direct comparison with simulated intensities from frozen-lattice multislice simulations [1,3]. The simulations included static atomic displacements, as well as the increase in the unit cell size and the Debye-Waller factor of Ga due to alloying with Sb. For pure zinc blende (ZB) and wurtzite (WZ) GaAs segments and GaAsSb segments near the catalytic droplet experiments and simulations displayed a nearly perfect fit indicating a radially homogeneous composition (Fig. 2(a)).

In the case of GaAsSb NWs, an increased reduction of $\mathrm{Sb}$ at the surface with increasing growth time was observed. In the HAADF STEM intensity profiles across the NWs this was observed as an increasing deviation from the simulated intensity profiles assuming a homogeneous radial $\mathrm{Sb}$ distribution when moving from the tip towards the bottom of the NW. The experimental HAADF STEM profiles could be explained using a diffusion model with endless Sb sink at the outer surface due to As-Sb exchange during growth (Fig. 2(b)).

In the case of the GaAsSb inserts an even more pronounced deviation from the simulated intensity profile assuming a homogeneous $\mathrm{Sb}$ distribution was observed. This could be explained by a combined effect of radial GaAs overgrowth and diffusion of Sb into the overgrown GaAs layer (Fig. 2(c,d)). The radial distribution profiles are different for the $\mathrm{Ga}$ - and Au-assisted growth as the growth temperatures are different. 
As the initial HAADF simulations assumed a homogeneous $\mathrm{Sb}$ distribution in the direction of the electron beam, the presence of a reduced $\mathrm{Sb}$ content towards the outer surface might have led to an underestimation of the $\mathrm{Sb}$ content based on quantitative STEM. Using different diffusion models developed for these hexagonal-shaped nanowires for the different growth scenarios applied, the maximum $\mathrm{Sb}$ concentration $\left(\mathrm{C}_{0}\right)$ in the core of the NW could be estimated (Fig. 2). These deducted maximum $\mathrm{Sb}$ concentrations were compared to the initial quantitative STEM results and energy dispersive spectroscopy measurements on the same NWs. Tentative intensity simulations based on inhomogeneous $\mathrm{Sb}$ profiles were performed to estimate the inaccuracy. The effect of the inhomogeneous radial Sb distributions on the optoelectronic properties is discussed.

\section{References:}

[1] H Kauko et al, Micron, 44 (2013), p. 254.

[2] J Todorovic et al, Semiconductor Science and Technology, 28 (2013), 115004.

[3] A Rosenauer and M Schowalter, Springer Proceedings in Physics, 120 (2008), p. 170.

[4] The authors acknowledge funding from Faculty of Natural Sciences and Technology at NTNU, and the "RENERGI" program of the Research Council of Norway under grant no. 190871 and the Deutsche Forschungsgemeinschaft under grant no. RO 2057/8-1.
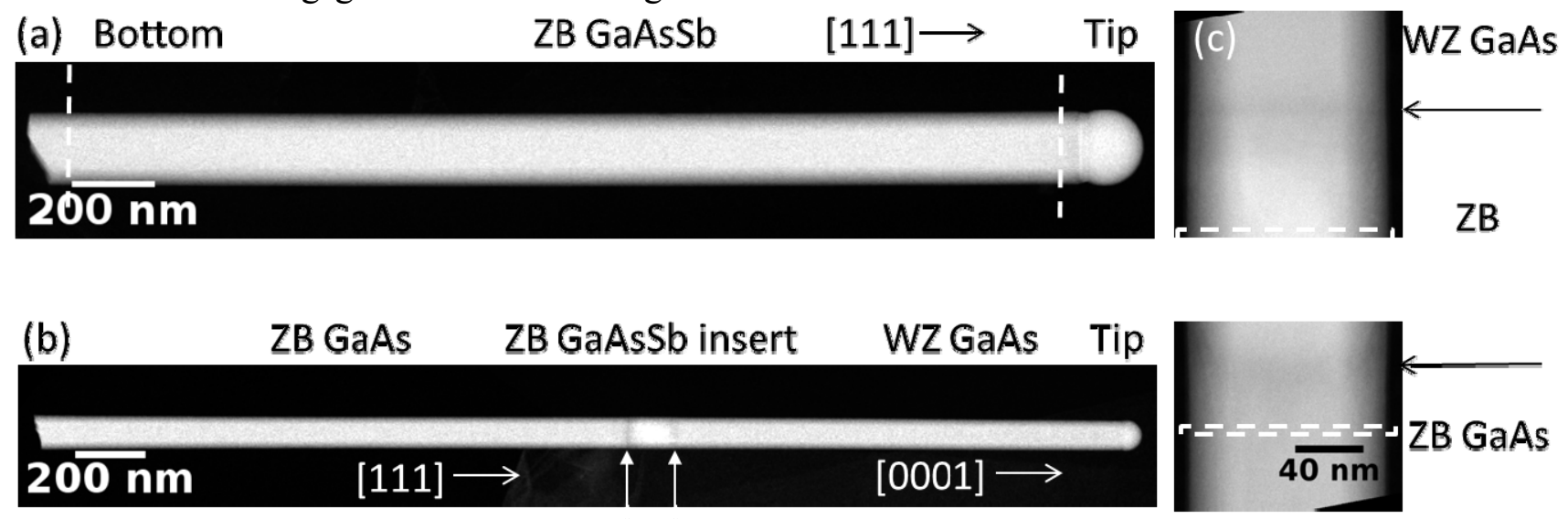

Figure 1. HAADF STEM images of (a) a Ga-assisted GaAsSb NW, (b) a Ga-assisted NW with $\mathrm{GaAsSb}$ insert, indicated by arrows, and (c) the insert in (b) at a higher magnification. Profiles across $\mathrm{ZB} \mathrm{GaAs}$ and GaAsSb are marked by dashed lines and boxes.

${ }_{0.35}$ (a) Pure WZ GaAs

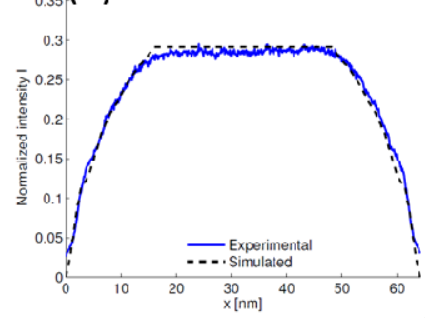

(b)ZB GaAs Sb bottom

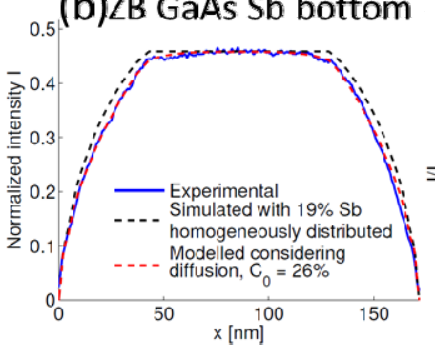

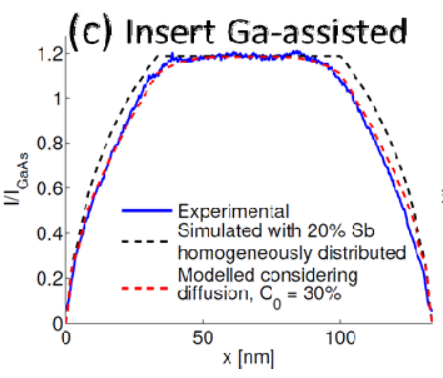

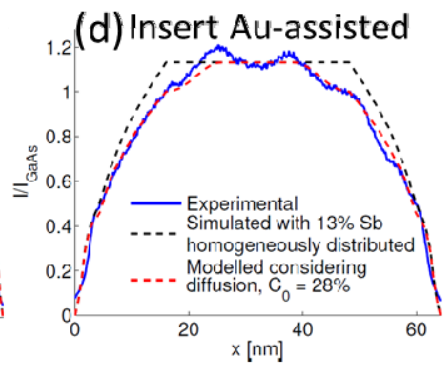

Figure 2. Example intensity profiles of (a) pure WZ GaAs reference region, (b) at the bottom of a $\mathrm{GaAsSb} \mathrm{NW}$, (c) at the center of a GaAsSb insert in Ga-assisted NW and (d) at center of a GaAsSb insert in Au-assisted NW. Blue is the experimental intensity profile, black dashed line is the simulated profile (assuming homogeneous $\mathrm{Sb}$ distribution) and red dashed line is the modelled profile, taking diffusion (b-d) and GaAs overgrowth $(c, d)$ into account. The profiles are normalized to the electron beam $(a, b)$ or GaAs reference area $(c, d)$. 